\title{
Protección de los niveles sociales y Recomendación de la OIT No 202
}

Protection Social Levels and ILO Recommendation Number 202

Níveis de proteção social e recomendação da OIT número 202

\author{
Jose Luis Dodera ${ }^{1}$ \\ ${ }^{1}$ Universidad del Trabajo del Uruguay \\ Orcid: https://orcid.org/0000-0002-8244-2333
}

Para citación: José Luis Dodera, Protección de los niveles sociales y Recomendación de la OIT $N^{o} 202$, DOI: https://doi.org/10.22235/rd.vi20.1857, Revista de Derecho N. 20 (2-2019): 11-24. ISSN 1510-3714, ISSN on line 2393-6193.

\section{Resumen:}

A partir de la aprobación de la Recomendación 202 (en adelante, R 202), por la Conferencia General del Trabajo, de la OIT en el año 2012, dirigida a los países miembros de la organización para la aprobación de medidas para construir pisos de protección social, dentro del marco de trabajo decente, entendemos que surgieron obligaciones para Uruguay, donde se pueden delinear dos ejes de trabajo básicos: a) la emersión del trabajo informal, no declarado o no registrado, la transición del trabajo informal, no declarado o no registrado hacia el trabajo formal, y b) la promoción del trabajo productivo y del empleo. En estos dos ejes, las cooperativas de trabajo y las cooperativas de producción constituyen instrumentos idóneos y tienen un papel relevante para completar la construcción del piso social.

Palabras clave: cooperativa de trabajo, cooperativa de producción, derecho laboral, Uruguay.

\section{Abstract:}

Since the adoption of Recommendation 202 (hereinafter, R 202), by the General Labour Conference of the ILO in 2012, addressed to the member countries of the organization for the adoption of measures to build floors protection social, within the framework of 
decent work, we understand that arose obligations to Uruguay, where you can delineate two axes of basic work: a) the emergence of informal work, undeclared or unregistered, the transition of informal work, undeclared or not registered to formal work, and b) the promotion of productive work and employment. In these two axes s, worker cooperatives and production cooperatives are the instruments and play an important role to complete the construction of the social floor.

Keywords: worker cooperative, production cooperative, Uruguay.

\section{Resumo:}

A partir da adopção da recomendação 202 (a seguir designada R 202), pela Conferência geral do trabalho da OIT em 2012, destinada aos países membros da organização para a adopção de medidas de construção de pavimentos de proteção social, dentro do quadro digno de trabalho, entendemos que as obrigações surgiram para o Uruguai, onde dois eixos básicos de trabalho podem ser delineados: (a) a emersão do trabalho informal, não declarada ou não registrada, a transição do trabalho informal, não declarada ou não para o trabalho formal, e (b) a promoção do trabalho produtivo e do emprego. Nestes dois eixos, as cooperativas de trabalho e cooperativas de produção são instrumentos adequados e têm um papel relevante a desempenhar na conclusão da construção do pavimento social. Palavras-chave: cooperativa, cooperativa de produção, direito do trabalho, Uruguai.

Sumario: I. Los pisos de protección. II. Ejemplos destacados de construcción de piso social. III. Acciones pendientes en la construcción del piso social. IV. Conclusión. Bibliografía.

\section{Los pisos de protección}

¿A qué nos referimos cuando hablamos de la construcción de los pisos de protección social? Cuando hablamos de pisos de protección social nos estamos refiriendo a la Seguridad Social y ampliar su cobertura de forma universal, cubriendo riesgos sociales de forma universal.

La R202, en su numeral 2, concibe a los pisos de protección social como "conjuntos de garantías básicas de seguridad social definidos a nivel nacional que aseguran una 
protección destinada a prevenir o a aliviar la pobreza, la vulnerabilidad y la exclusión social"

El preámbulo de la R 202, reafirma a la Seguridad Social como un derecho humano fundamental. Esta concepción y reafirmación tiene su fuente normativa en los artículos 22 y 25 de la Declaración Universal de Derechos Humanos, recibiendo también su reconocimiento como derecho económico y social por el Pacto de los Derechos Económicos, Sociales y Culturales, en sus artículos 9,11 y 12.

En el preámbulo de la Recomendación 202 se reconoce a la Seguridad Social, junto a la promoción del empleo como instrumento útil para el desarrollo y progreso, "como una herramienta para prevenir y reducir la pobreza, la desigualdad, la exclusión social y la inseguridad social, para promover la igualdad de oportunidades, la igualdad de género y la igualdad racial y para apoyar la transición del empleo informal al empleo formal"2.

La Seguridad Social es visualizada por la OIT como una inversión en las personas y que los sistemas de seguridad social actúan como estabilizadores sociales y económicos, facilitando una transición hacia una economía sostenible ${ }^{3}$.

Respecto a la Seguridad Social, también podemos citar otras normas como son: "C102 Convenio sobre la seguridad social (norma mínima), 1952 (núm. 102) R 67 - Recomendación sobre la seguridad de los medios de vida, 1944 (núm. 67) (12 mayo 1944). R 69 Recomendación sobre la asistencia médica, 1944."

Así mismo, la Declaración de la OIT sobre la Justicia social para una globalización equitativa reconoce que "los compromisos y esfuerzos de los Miembros y de la Organización para poner en práctica al mandato constitucional de la OIT, en particular mediante las normas internacionales del trabajo, y para situar al empleo pleno y productivo y el Trabajo Decente como elemento central de las políticas económicas y social, deberían basarse en (el objetivo de) ii adoptar y ampliar medidas de protección (...) que sean sostenibles y estén adaptados a las circunstancias nacionales, con inclusión de la ampliación de la Seguridad Social a todas las personas."

Estas normas ponen en práctica al mandato constitucional de la OIT, situando al empleo pleno y productivo y al Trabajo Decente como elemento central de las políticas económicas y sociales, deberían basarse en (el objetivo de) ii) adoptar y ampliar medidas de protección (...)

${ }^{2}$ http://www.ilo.org/dyn/normlex/es/f?p=NORMLEXPUB:12100:0::NO::P12100_ILO_

CODE:R202 Recomendación N 202, Preámbulo. 4to Párrafo.

${ }^{3}$ Recomendación N²02, Preámbulo. 4to Párrafo. 
que sean sostenibles y estén adaptados a las circunstancias nacionales, con inclusión de la ampliación de la seguridad social a todas las personas" 4

La Recomendación sobre los pisos de protección social, 2012 (núm. 202) surgió como una necesidad para complementar las normas existentes en materia de Seguridad Social, cumpliendo además una función de orientación a los Miembros en procura de establecer pisos de protección social adaptados a las circunstancias y niveles de desarrollo de cada país, como parte de sistemas integrales de seguridad social" 5 (las letras destacadas son nuestras).

A los efectos de esta ponencia, nos concentramos en algunos aspectos de la R202:

a. los trabajos a nivel preparatorio, las actas provisionales “...a priorización de políticas destinadas a promover un crecimiento sostenible a largo plazo, asociadas a la inclusión social, contribuye a superar la pobreza extrema y a reducir las desigualdades y diferencias sociales... reconociendo que la transición al empleo formal y el establecimiento de sistemas de seguridad social sostenibles se refuerzan mutuamente" 6

b. Capítulo I de la R 202: Objetivos, Ámbito de Aplicación y Principios: Los pisos de protección tienen el propósito de constituirse como un elemento fundamental en los sistemas nacionales de seguridad social.

Los principios enunciados en la R 202, orientan al país Miembro de la OIT a extender los pisos de protección hacia sectores no alcanzados de la seguridad social, dentro del marco de trabajo decente - sin discriminación, igualdad de trato, promoción de la protección social, por un empleo libre y productivo, en libertad sindical, y bajo los principios : universalidad, de acuerdo a lo prescripto en la legislación nacional, adecuación y previsibilidad de las prestaciones, sin discriminación, inclusión social, respeto a la persona beneficiaria, realización progresiva, solidaridad, gestión financiera, sana y transparente, sostenibilidad financiera, coherencia con las políticas

4 www.ilo.org. Actas Provisionales 101 reunión, Ginebra, mayo - junio 2012 Documento 14 A, pág.14 A/2

${ }^{5}$ Actas Provisionales 101.

${ }^{6}$ Actas Provisionales 101, pàg. 14/A/1 y 8vo párrafo del Preámbulo de la R202. 
sociales, económicas y de empleo, participación tripartita, seguimiento regular de la aplicación y evaluación periódica, etc. (artículo 3ro) ${ }^{7}$.

c. Ámbito de Acción Local: En el Capítulo II, "Pisos Nacionales de Protección Social", a los efectos de este trabajo destacamos dos literales como rectores para nuestro país, transcribiéndolos a ambos a continuación. "b) numeral 10 de la recomendación, orienta al Miembro de la OIT, entre otras cosas, a promover la actividad económica productiva y al empleo formal, considerando la adopción de políticas que incluyen la contratación pública, la concesión de créditos públicos, la inspección del trabajo, políticas del mercado de trabajo e incentivos fiscales y que fomentan la educación, la formación profesional, las competencias productivas y la empleabilidad”. “c) asegurar la coordinación con otras políticas que fomentan el empleo formal, la generación de ingresos, la educación, la alfabetización, la formación profesional, las competencias y la empleabilidad, que reducen la precariedad, y que promueven el trabajo estable, la iniciativa empresarial y las empresas sostenibles en un marco de trabajo decente" 8

Los ejes de acción que resaltamos como necesarios para lograr más avances en concretar los Pisos Nacionales de Protección Social, nos concentramos en la promoción de la actividad económica productiva y al empleo formal, surgiendo como objetivos propios el fomento, protección y desarrollo de las formas cooperativas, en especial a las formas cooperativas de producción y de trabajo y a la emersión del trabajo no declarado, no registrado, sumergido.

Esta línea de trabajo es resaltada de forma más explícita por las Actas Provisionales, actas preparatorias de la Recomendación 202, donde en el numeral 14 literal d) señala: “complementar la seguridad social con políticas activas del mercado de trabajo, inclusive mediante formación profesional u otras medidas, según proceda" 9

${ }^{7}$ http://www.ilo.org/dyn/normlex/es/f?p=NORMLEXPUB:12100:0::NO::P12100_ILO CODE:R202 Recomendación $N^{\circ}$ 202. Capítulo I. Objetivos, Ámbito de Aplicación y Principios.

${ }^{8}$ http://www.ilo.org/dyn/normlex/es/f?p=NORMLEXPUB:12100:0::NO::P12100_ILO CODE:R202 Recomendación $N^{\circ}$ 202. Capítulo II. Pisos Nacionales de Protección Social"

${ }^{9}$ Actas Provisionales $101^{\circ}$ reunión, Ginebra, mayo - junio 2012 Documento 14 A, Pág. $14 \mathrm{~A} / 5$ 
Para lograr alcanzar ambos objetivos, importa destacar la experiencia comparada de Italia, con la ley 383/2011, a través de la cual, se propuso a los agentes económicos una gradual formalización de los trabajadores no declarados, sumergidos o no registrados, herramienta que se suma al instrumento comunitario, Pacto Territorial -donde todos los actores de un área geográfica determinada pactan objetivos productivos y sociales dentro de un marco de diálogo social-

De intentar aplicar en alguna medida similar propósito en nuestro país, resulta estratégico un mayor impulso del sistema cooperativo por dos razones fundamentales, es una figura esencialmente colectiva y muestra una gran ductilidad a la hora de implementar soluciones.

En vistas a la puesta en práctica, es necesario identificar a las actividades económicas y a las áreas geográficas donde se registra al trabajo sumergido, no declarado o no registrado, e implementar un plan de trabajo y quinquenal.

La emersión o formalización del trabajo sumergido constituye una oportunidad para proteger, y garantizar derechos, crear puestos de trabajo y lograr la sostenibilidad, en un marco de igualdad de género.

La construcción del piso social contiene ejes de acción como son la incorporación de las diferentes actividades informales a la seguridad social, la inclusión de estos colectivos a los regímenes universales de seguridad social, a establecer diversos regímenes de asistencia social por categorías de beneficiarios - de acuerdo con los riesgos sociales cubiertos-, la construcción del seguro social basado en el empleo; en suma, que el seguro social sea básicamente un sistema contributivo ${ }^{10}$.

Conviene recordar la concepción de seguridad social de la O.I.T, "la noción de seguridad social adoptada comprende todas las medidas que conceden prestaciones, ya sea en especie, para garantizar la protección en caso de, entre otras cosas, a) una falta de ingresos relacionados con el trabajo (o ingresos insuficientes) ocasionados por una enfermedad, discapacidad, maternidad, los accidentes laborales y las enfermedades profesionales, el desempleo, la vejez o el fallecimiento de un miembro de familia, b) una falta de acceso o un acceso inasequible o la asistencia médica, c) un apoyo familiar insuficiente,

${ }^{10}$ Ampliación de la Protección Social. OIT extensión de la Cobertura de Seguridad Social a la Economía Informal. Archivo digital ubicado en www.ilo.org., 2. 
especialmente en el caso de los hijos mayores de los adultos dependientes, d) la pobreza general y la exclusión social"11

De lo cual surge que la seguridad social comprende dos dimensiones principales, "la seguridad del ingreso (Recomendación 67 y Recomendación 69, ambas de la OIT), la disponibilidad de la asistencia médica.

La noción de seguridad social aportada está en línea con lo establecido en la Declaración de Filadelfia, "las medidas de seguridad social para garantizar ingresos básicos o quienes necesiten y prestar asistencia médica completa" ${ }^{\prime 2}$.

El acceso a la seguridad social es, en su naturaleza esencial, una responsabilidad pública, y se otorga clásicamente a través de instituciones públicas, por más que pudiere delegarse a entidades o instituciones privadas y que éstas asumieran algunas funciones propias de la seguridad social como son la recepción de cotizaciones y la prestación de servicios. ${ }^{13}$

El documento de referencia en este punto, “Ampliación de la Protección Social. Extensión de la cobertura de Seguridad Social a la Economía Informal" ${ }^{14}$ contiene dos ideas que son angulares, la primera es que "la falta de protección social agrava considerablemente la exclusión social y la pobreza ${ }^{15}$, mientras que la segunda es "la necesidad de comprender los factores determinantes de la falta de protección social en la economía informal es esencial para formular soluciones de política que amplíen la cobertura". La informalización del trabajo amenaza la existencia del seguro social universal. La gobernanza y la eficiencia del seguro social deben ser consideradas como una política necesaria para reducir la informalización y la inclusión de los colectivos marginados ${ }^{16}$. La gobernanza del sistema de seguridad social necesariamente tendrá como principios rectores a la universalización y a la inclusión de los colectivos de la sociedad, como su razón de ser. ${ }^{17}$

\footnotetext{
${ }^{11}$ Ampliación de la Protección Social, 2.

12 Ampliación de la Protección Social, 2.

${ }^{13}$ Ampliación de la Protección Social, 3.

${ }^{14}$ Ampliación de la Protección Social, 2.

${ }^{15}$ Ampliación de la Protección Social, 2.

${ }^{16}$ Ampliación de la Protección Social, 4 y 5.

${ }^{17}$ Ampliación de la Protección Social, 4 y 5.
} 
Los nuevos enfoques y las buenas prácticas en la Seguridad Social reseñadas por la OIT en el documento de referencia tiene como puntos: concebir a la Seguridad Social como un derecho, tener en cuenta a la diversidad, ampliar la cobertura del seguro social basado en el empleo, establecer un seguro de salud nacional, poner en marcha regímenes no contributivos financiados mediante impuestos, combinar instrumentos de política en el contexto de una estrategia de Seguridad Social nacional integrada (las letras destacadas son nuestras $)^{18}$.

\section{Ejemplos destacados de construcción de piso social}

Dentro de las experiencias exitosas de construcción de piso social, relevadas por la OIT, se cita al programa Bolsa Familia (Brasil), al Monotributo (Uruguay), identificamos y subrayamos la experiencia del Sistema Nacional del Empleo Rural (NREGS) de la India $(2005)^{19}$, a través del cual un hogar rural tiene derecho a solicitar 100 jornales de empleo manual no calificado al año y se reserva un cupo de un tercio de esas oportunidades de empleo a mujeres. A través de este programa, se ponen en práctica proyectos de gestión de recursos hídricos, de la gestión de la tierra, de obras de infraestructura.

La coordinación y coherencia de políticas entre los diversos mecanismos de seguridad social permite aprovechar al máximo los recursos para evitar la exclusión y promover la salida de la informalidad ${ }^{20}$.

\footnotetext{
${ }^{18}$ Ampliación de la Protección Social, 4 y 5.

${ }^{19}$ Ampliación de la Protección Social, 12.

${ }^{20}$ Ampliación de la Protección Social, 14.
} 
En Argentina, obtuvo un avance en el combate al empleo no registrado (ENR) a partir de un enfoque de políticas públicas, integrando y articulando programas y acciones sociolaborales, económicas ${ }^{21.22}$

En Uruguay, en los últimos quince años, en procura de la construcción del Piso de Seguridad Social ${ }^{23}$, destacamos:

a) hubo un cambio en la definición de los beneficiarios de las asignaciones Familiares, pasando de ser un beneficio social de naturaleza contributiva a constituirse a un beneficio no contributivo, no requiriéndose más aportes previos para acceder al mismo,

b) la creación del Monotributo - un único tributo que cubre las contribuciones sociales a la seguridad social (seguro de invalidez, vejez, seguro de enfermedad) así como la contribución y pago de los impuestos nacionales - una forma simple y mínima de cotización a la seguridad social destinada a sectores con mayor incidencia del trabajo informal,

c) la implementación del Plan de Equidad, programa social destinado a sectores vulnerables de la sociedad, con acciones de capacitación, subsidio económico, y cotización a la seguridad social,

d) la convocatoria a los Consejos de Salarios (mecanismo administrativo de fijación tripartito de los salarios mínimos y categorías) por parte del Ministerio de Trabajo y Seguridad Social, donde se promueve la negociación colectiva. El Consejo de Salarios alcanza a casi el $100 \%$ de las actividades económicas.

${ }^{21}$ Bertranou, Fabio, "La Formalización laboral en Argentina: Avances recientes y el camino por recorrer", Véase https://www.academia.edu/4996820/La_formalizaci\%C3\%B3n_laboral_en_Argentina Avances_recientes_y_el_camino_por_recorrer. Captura de pantalla 25 de marzo de 2019, 7.38 horas.

${ }^{22}$ CAMPOS, M y GALÍN, P. (2006), "La Experiencia Internacional sobre Políticas para la Regularización del Empleo No Registrado", www.trabajo.gov.ar, archivo recuperado www.trabajo.gov.ar/left/estadisticas/descargas/.../2006n02_a10_mCampos.pdf. Captura de pantalla: 13 de mayo de 2018 hora 08.30.

${ }^{23}$ Véase: http://www.cuestaduarte.org.uy/investigacion/economia/otrosdocumentos/item/61-formalizacion-del-empleo-en-uruguay-2004-2012. Captura de pantalla 25 de marzo de 2019, 07.30 horas. 
e) regulación específica del trabajo doméstico, uno de los sectores económicos donde se concentra el trabajo informal ${ }^{24}$,

f) instancias de Diálogo Social, consultas de carácter tripartito, que produjeron productos concretos, produciéndose modificaciones en la legislación, produciéndose una flexibilización de los requisitos para acceder a las prestaciones, se redujeron exigencias para hacer posible el acceso a los beneficiarios. Al respecto, en primer lugar, se contempló la situación de la mujer madre de hijos, quien se quedó a cargo de la crianza de los niños en sus primeros años, computando una contribución ficta de un año de trabajo por cada hijo con un máximo de dos años de trabajo, aunque tuviese dos o más hijos, a los efectos del cómputo de los años de servicio requeridos como mínimos años de servicio.

En segundo lugar, se redujo el mínimo de años de servicio reconocidos, de 35 años pasó a ser 30 años el mínimo de años de servicios reconocidos.

En tercer lugar, se creó un subsidio de desocupación forzada especial para aquellos trabajadores con veintiocho (28) años de servicios reconocidos por el ente de previsión social y tener cincuenta y ocho (58) años, a los efectos de que a través del subsidio logren alcanzar la edad mínima de trabajo y el mínimo de los años de servicios reconocidos exigidos. La duración máxima del subsidio especial es de dos años y justamente estos dos años son los que permiten al beneficio completar los 30 años de servicios reconocidos como mínimo.

En cuarto lugar, se modificó el seguro de desempleo o de paro, produciéndose dos variantes significativas que son: la ampliación del plazo de cobertura para los trabajadores mayores de 50 años por ser trabajadores con mayores dificultades de obtener un empleo dentro del mercado de trabajo y la cobertura del seguro de paro o desocupación a aquellos trabajadores que hayan perdido a su principal empleo, haciendo compatible el cobro del subsidio de desempleo con la continuidad en el trabajo en el empleo secundario o de menor ingreso en el período previo al envío al seguro de paro.

En quinto lugar, la ampliación de la Cobertura de la Licencia por Maternidad extendida a ambos progenitores, ampliándose la cobertura también a trabajadores independientes, microempresarios, así como la ampliación del plazo de su cobertura, pasando de 12 a 14 meses.

${ }^{24}$ Loustanau, Nelson, Trabajo Doméstico (Montevideo: F.C.U., 2013). 
En quinto lugar, la implementación de la Tarjeta de Alimentación, por la cual se garantiza la subsistencia a las personas en situación de extrema pobreza a través de la provisión de alimentos en especie.

En sexto lugar, la implementación del Fondo Nacional de Salud como un sistema de provisión de fondos a emprendimientos colectivos.

En séptimo lugar, la implementación del Sistema Integrado de Salud, por la cual se garantiza el primer nivel de salud, asistencia primaria y preventiva, a la población.

En octavo lugar, la implementación de medidas de promoción de microemprendimientos con la instalación de servicios de asesoramiento en su constitución y líneas de crédito reservadas a estos fines,

En noveno lugar, la creación de modalidades de figuras cooperativas como son las cooperativas de trabajo y cooperativas sociales, por la aprobación de la ley 18.407, destacando simultáneamente que esta norma permite a las cooperativas de producción y/o de trabajo, participar en forma conjunta, colectiva, asociada con otros agentes económicos, ya sea en grupos o individualmente.

En décimo lugar, la creación de Fondo Estructural (Fon.des), constituido con fondos provenientes del diez por ciento de las utilidades del banco público y destinado a financiar emprendimientos productivos cooperativos.

En décimo primer lugar, la reserva de cuota a ingresos en cargos públicos a personas afrodescendientes por la aprobación de la ley de cuotas de afrodescendientes.

En décimo segundo lugar, también hay que destacar a la ley 19.292, donde existe un régimen de compras estatales que beneficia a Organizaciones de Producción Familiar Agropecuaria y de la pesca artesanal, estableciéndose una reserva de mercado mínima del $30 \%$ para las compras no centralizadas, de bienes alimentarios, alcanzándose a los productos artesanales agropecuarios en su estado natural, los productos artesanales agropecuarios y los productos de la pesca artesanal, ingresando también los alimentos procesados elaborados con materia prima de los productores integrantes de las organizaciones familiares habilitadas. Se considera Organización Habilitada a toda aquella que esté integrada al menos por cinco productores agropecuarios y de los cuales, al menos el $70 \%$ deben ser productores familiares agropecuarios y/o pescadores artesanales" 25 .

${ }^{25}$ Ley 19.292, artículo 5to. 


\section{Acciones pendientes en la construcción del piso social}

Visualizamos la emersión del trabajo sumergido o no declarado como materia pendiente para la construcción del Piso Social y huelgan las acciones de promoción del empleo pleno y productivo.

A partir de los datos que citamos a continuación, provenientes del Instituto Nacional de Estadísticas, observamos que la concentración del trabajo sumergido sucede en centros poblados donde existe menor población ${ }^{26}$, en lugares alejados de Montevideo (la capital del Uruguay $)^{27}$, en personas jóvenes - acentuándose en menores de 20 años (75\% de estos jóvenes), persistiendo la concentración de la actividad no declarada hasta los treinta años (39,1\% de estos últimos jóvenes) $-^{28}$ y en mayores de 65 años (63,2 \% de los adultos), en unidades productivas que son micro y pequeñas empresas (en unipersonales llega al $73,1 \%$, en microemprendimientos alcanza al 48,4\%), ${ }^{29}$ en actividades que se realizan en su vivienda o en la calle en puesto fijo, en servicios de comercio al por mayor o por menor, en servicios destinados a automotores, motocicletas, en hoteles y restaurantes (45\%), en actividades de desperdicios, aguas residuales, saneamiento o en actividades similares $(51 \%)$ y en el servicio doméstico $(63 \%)^{30}$.

Estos datos suministrados demuestran las acciones por donde las acciones públicas deben emprenderse, acciones a emprender en zonas alejadas de la capital, en centros poblados con menores de 5.000 habitantes, en aquellas personas que comienzan a trabajar y en aquellas personas que teniendo edad para el retiro no lo logran obtener y continúan

${ }^{26}$ Empleo Informal en el Uruguay. Encuesta Nacional de Hogares. 2006. Instituto Nacional de Estadìsticas.2006. Versión Digital. Publicado en la página web: www.ine.gub.uy Cuadro Numero3. Página 6.

27 Empleo Informal en el Uruguay. Encuesta Nacional de Hogares. 2006. Instituto Nacional de Estadìsticas.2006. Versión Digital. Publicado en la página web: www.ine.gub.uy Cuadro Numero2. Página 6.

${ }^{28}$ Empleo Informal en el Uruguay. Cuadro Numero 8. Página 5.

${ }^{29}$ Empleo Informal en el Uruguay. Cuadro Numero 4. Página 7.

${ }^{30}$ Empleo Informal en el Uruguay. Cuadro Numero 6 Página 9. 
trabajando en forma sumergida, en actividades de servicio doméstico y en actividades relacionadas con los desperdicios, o sea con los recolectores de basura.

\section{Conclusión}

A partir de la aprobación de la Recomendación 202 (R 202), por la Conferencia General del Trabajo, de la OIT en el año 2012, dentro del marco de trabajo decente, surgieron obligaciones para Uruguay, donde se pueden delinear dos ejes de trabajo básicos: a) la emersión del trabajo informal, no declarado o no registrado, la transición del trabajo informal, no declarado o no registrado hacia el trabajo formal, y b) la promoción del trabajo productivo y del empleo. En estos dos ejes, las cooperativas de trabajo y las cooperativas de producción constituyen instrumentos idóneos y tienen un papel relevante para completar la construcción del piso social.

En el primer eje, la emersión del trabajo informal ha avanzado Uruguay, teniendo actualmente aún trabajo informal para formalizar, mientras que, en el segundo eje, en la promoción del trabajo productivo y del empleo, se promovió la forma cooperativa con acciones dirigidas por el Estado.

Ahora, el interés de fomentar la forma cooperativa es lógica porque la forma cooperativa es por esencia una solución conjunta de personas que se unen para lograr un sustento y una forma de gestión del medio de producción.

La flexibilidad y la autoregulación constituyen puntos de fuerza y también constituyen puntos débiles de esta forma de organización.

Las acciones públicas deben conducir al fortalecimiento de estas soluciones colectivas, como una medida más para la construcción del piso social recomendado por la OIT, en su recomendación Número 202, ya que son una alternativa a la exclusión social.

Las cooperativas también son un instrumento para alcanzar las metas del trabajo decente (empleo productivo, con inclusión social, libremente elegido, con protección social y sin discriminación, en cumplimiento de la normativa tanto individual como colectiva).

Contribución: $100 \%$ del autor

Bibliografía 
Bertranou, Fabio, "La Formalización laboral en Argentina: Avances recientes y el camino por recorrer",

www.academia.edu. $/ 4996820 / \mathrm{La}$ _formalizaci\%C3\%B3n_laboral_en_Argentina_Avanc es_recientes_y_el_camino_por_recorrer.

Campos, M y Galín, P, “La Experiencia Internacional sobre Políticas para la Regularización del Empleo No Registrado", www.trabajo.gov.ar, archivo recuperado www.trabajo.gov.ar/left/estadisticas/descargas/.../2006n02_a10_mCampos.pdf.

Empleo Informal en Uruguay Encuesta Nacional de Hogares 2006. Instituto Nacional de Estadística 2006. Versión Digital, publicado en la página web www.ine.gub.uy. Loustanau, Nelson, Trabajo Doméstico, Montevideo: F.C.U, 2013.

www.ilo.org. Actas Provisionales 101 Reunión, Ginebra, mayo - junio 2012, Documento $14 \mathrm{~A}$.

www.ilo.org. Recomendación 202. 